Parallel Processing Letters, Vol. 11, No. 4 (2001) 503-505

(C) World Scientific Publishing Company

\title{
AUTHOR INDEX \\ VOLUME 11 (2001)
}

Akl, S. G., see Bruda, S. D.

Amaral, J. N., see Kakulavarapu, P.

11 (2001) 353-361

11 (2001) 169-184

Amamiya, M., Taniguchi, H. and Matsuzaki, T., An architecture of fusing communication and execution for global distributed processing

Anglano, C., see Chiola, G.

11 (2001) 7-24

11 (2001) 223-236

Arantes, L. Poitrenaud, D., Sens, P. and Folliot, B., The barrier-lock clock: A scalable synchronization-oriented logical clock

$11(2001) 65-76$

Arnold, D. C., Vahdiyar, S. S. and Dongarra, J. J., On the convergence of computational and data grids

Baden, S. B., see Kelly, P. H. J.

Baker, M. and Smith, G., Establishing a reliable Jini infrastructure for parallel applications

Beckmann, O., see Kelly, P. H. J.

Bruda, S. D. and Akl, S. G., On the necessity of formal models for real-time parallel computations

Butz, C., see Loidl, H.-W.

Cérin, C. and Gaudiot, J.-L., Benchmarking clusters of workstations through parallel sorting and BSP libraries

Castillo, L., see Siegelin, C.

Chen, Y. and Sanders, J. W., Weakest specifunctions for BSP

Chiola, G., Ciaccio, G. and Anglano, C., On the use of coscheduling in fast communications systems

Christodorescu, M., see Miller, B. P.

Ciaccio, G., see Chiola, G.

Cosnard, M. and Jeannot, E., Automatic parallelization techniques based on compact DAG extraction and symbolic scheduling

Cosnard, M., Editorial note

Danelutto, M., Efficient support for skeletons on workstation clusters

11 (2001) 187-202

11 (2001) 455-470

11 (2001) 203-221

11 (2001) 455-470

11 (2001) 353-361

11 (2001) 471-486

$11(2001) 25-40$

11 (2001) 57-64

11 (2001) 439-454

11 (2001) 223-236

11 (2001) 267-280

11 (2001) 223-236

11 (2001) 151-168

$11(2001) 311$

11 (2001) 41-56

Diallo, M., Ferreira, A. and Rau-Chaplin, A., A note on communication-efficient deterministic parallel algorithms for planar point location and 2D Voronoï Diagram

11 (2001) 327-340

Dongarra, J. and Tourancheau, B., Preface: Clusters and computational grids for scientific computing

$11(2001) 185$

Dongarra, J. J., see Arnold, D. C.

11 (2001) 187-202 
Evripidou, P., Samaras, G., Spyrou, C. and Papagapiou, A., Net-console: Web-based development environment for parallel programs

11 (2001) 139-150

Ferreira, A., see Diallo, M.

Field, T., see Kelly, P. H. J.

Finger, U., see Siegelin, C.

Folliot, B., see Arantes, L.

Gao, G. R., see Kakulavarapu, P.

Gaudiot, J.-L., see Cérin, C.

Gautama, H., see Reijns, G. L.

Geoffray, P., OPIOM: Off-processor IO with Myrinet

Guo, M., Denotational semantics of an HPF-like data-parallel language model

11 (2001) $327-340$

11 (2001) 455-470

11 (2001) 57-64

11 (2001) 65-76

11 (2001) 169-184

11 (2001) 25-40

11 (2001) 109-123

11 (2001) 237-250

Habbas, Z., Krajecki, M. and Singer, D., Shared memory implementation of constraint satisfaction problem resolution

11 (2001) 363-374

11 (2001) 487-501

Hains, G. and Miller, Q., Preface: High-level parallel programming and applications

Hsu, W.-J., see Vee, V.-Y.

Iverson, R., see Miller, B. P.

Jeannot, E., see Cosnard, M.

Kakulavarapu, P., Maquelin, O. C., Amaral, J. N. and Gao, G. R., Dynamic load balancers for a multithreaded multiprocessor system

Kelly, P. H. J., Beckmann, O., Field, T. and Baden, S. B., THEMIS: Component dependence metadata in adaptive parallel computations

Kosar, T., see Miller, B. P.

Krajecki, M., see Habbas, Z.

Kranzlmüller, D., Nondeterminism analysis on supercomputers and clusters

Lee, S., Park, C.-I. and Park, C.-M., An improved parallel algorithm for Delaunay triangulation on distributed memory parallel computers

11 (2001) 375-376

11 (2001) 313-325

11 (2001) 267-280

11 (2001) 151-168

11 (2001) 169-184

11 (2001) 455-470

11 (2001) 267-280

11 (2001) 487-501

11 (2001) 251-266

Loidl, H.-W., Trinder, P. W. and Butz, C., Tuning task granularity and data locality of data parallel GPH programs

Loulergue, F., Distributed evaluation of functional BSP programs

Maquelin, O. C., see Kakulavarapu, P.

Matsuzaki, T., see Amamiya, M.

Migliardi, M. and Sunderam, V., Emulating parallel programming environments in the harness metacomputing system

Miller, B. P., Christodorescu, M., Iverson, R., Kosar, T., Mirgorodskii, A. and Popovici, F., Playing inside the black box:

Using dynamic instrumentation to create security holes

11 (2001) 341-352

11 (2001) 471-486

11 (2001) 423-437

11 (2001) 169-184

11 (2001) 7-24

11 (2001) 281-295

Miller, Q., see Hains, G.

Mirgorodskii, A., see Miller, B. P.

Mongelli, H. and Song, S. W., Parallel pattern matching with scaling

Mostefaoui, A. and Raynal, M., Leader-based consensus

11 (2001) 267-280

11 (2001) 375-376

11 (2001) 267-280

11 (2001) 125-138

11 (2001) 95-107 
Papagapiou, A. see Evripidou, P.

Park, C.-I., see Lee, S.

Park, C.-M., see Lee, S.

Perrott, R., see Phillips, C.,

Phillips, C. and Perrott, R., Problems with data parallelism

Poitrenaud, D., see Arantes, L.

Popovici, F., see Miller, B. P.

Rau-Chaplin, A., see Diallo, M.

Raynal, M., see Mostefaoui, A.

Reijns, G. L., van Gemund, A. J. C. and Gautama, H., Performance analysis of multi-stage interconnection networks with deterministic service times

Samaras, G., see Evripidou, P.

Sanders, J. W., see Chen, Y.

Sens, P., see Arantes, L.

Serot, J., Tagged-token data-flow for skeletons

Siegelin, C., Castillo, L. and Finger, U., Smart cards:

Distributed computing with $\$ 5$ devices

Singer, D., see Habbas, Z.

Smith, G., see Baker, M.

Song, S. W., see Mongelli, H.

Spyrou, C., see Evripidou, P.

Sunderam, V., see Migliardi, M.

Taniguchi, H., see Amamiya, M.

Tiskin, A., A new way to divide and conquer

Tourancheau, B. and Westrelin, R., Study of the medium message performance of BIP/Myrinet

Tourancheau, B., see Dongarra, J.

Trinder, P. W., see Loidl, H.-W.

Vahdiyar, S. S., see Arnold, D. C.

van Gemund A. J. C., see Reijns, G. L.

Vee, V.-Y. and Hsu, W.-J., A scalable and efficient storage allocator on shared-memory multiprocessors

Westrelin, R., see Tourancheau, B.

Zavanella, A., Skeletons, BSP and performance portability
11 (2001) 139-150

11 (2001) 341-352

11 (2001) 341-352

11 (2001) 77-94

11 (2001) 77-94

11 (2001) 65-76

11 (2001) 267-280

11 (2001) 327-340

11 (2001) 95-107

11 (2001) 109-123

11 (2001) 139-150

11 (2001) 439-454

11 (2001) 65-76

11 (2001) 377-392

$11(2001) 57-64$

11 (2001) 487-501

11 (2001) 203-221

11 (2001) 125-138

11 (2001) 139-150

11 (2001) 281-295

11 (2001) 7-24

11 (2001) 409-422

11 (2001) 297-309

11 (2001) 185

11 (2001) 471-486

11 (2001) 187-202

11 (2001) 109-123

11 (2001) 313-325

11 (2001) 297-309

11 (2001) 393-407 